\title{
Designing the IShTAR antenna: Physics and engineering aspects
}

F. Louche' , J. Jacquot, K. Crombé, D. Van Eester, R. D'Inca, S. Devaux, E. Faudot, H. Faugel, H. Fünfgelder, S. Heuraux, I. Morgal, J. Moritz, R. Ochoukov, and J.-M. Noterdaeme

Citation: AIP Conference Proceedings 1689, 070016 (2015); doi: 10.1063/1.4936523

View online: http://dx.doi.org/10.1063/1.4936523

View Table of Contents: http://aip.scitation.org/toc/apc/1689/1

Published by the American Institute of Physics

Articles you may be interested in

Studies of RF sheaths and diagnostics on IShTAR

AIP Conference Proceedings 1689, 030006030006 (2015); 10.1063/1.4936471 


\title{
Designing the IShTAR antenna: physics and engineering aspects
}

\author{
F. Louche*,a), J. Jacquot ${ }^{\dagger}$, K. Crombé,***, D. Van Eester*, R. D’Inca ${ }^{\dagger}$, S. Devaux ${ }^{\ddagger}$, \\ E. Faudot ${ }^{\ddagger}$, H. Faugel ${ }^{\ddagger}$, H. Fünfgelder ${ }^{\dagger}$, S. Heuraux ${ }^{\ddagger}$, I. Morgal ${ }^{\dagger}$, J. Moritz ${ }^{\ddagger}$, \\ R. Ochoukov ${ }^{\dagger}$ and J.-M. Noterdaeme ${ }^{\dagger, * *}$ \\ *Laboratory for Plasma Physics, ERM/KMS, EUROfusion Consortium Member, Brussels, Belgium \\ ${ }^{\dagger}$ Max-Planck-Institut für Plasmaphysik, Garching, Germany \\ **Department of Applied Physics, Ghent University, Ghent, Belgium \\ †Institut Jean Lamour UMR 7198 CNRS-Université de Lorraine, Nancy, France \\ a)Corresponding author: fabrice.louche@rma.ac.be
}

\begin{abstract}
IShTAR (Ion cyclotron Sheath Test ARrangement) is a magnetised plasma test facility installed at the Max-PlanckInstitut für Plasmaphysik in Garching, Germany. The main purpose of this device is the study of RF sheaths generated in front of ICRF (Ion Cyclotron Range of Frequency) antennas in magnetically confined plasmas. The plasma is generated by a helical $\mathrm{RF}$ antenna potentially able to reach a helicon mode. We present in this work recent modelling activities dedicated to IShTAR. On the one hand a parameterized magnetostatic model of the magnetic configuration was created with the finite element solver COMSOL Multiphysics [3]. The model considers two non-axial sets of coils and notably reproduces the magnetic field lines deviation at the center of the main vessel and the ripples observed during experiments. From this model we can infer that $2.4 \mathrm{kA}$ are required in the 2 main large coils of IShTAR for $1 \mathrm{kA}$ in the 4 small coils to generate a "smooth" magnetic field along field lines. On the other hand an ICRF antenna has been designed for IShTAR. A tridimensional model of the IShTAR vessel was developed with the electromagnetic code MicroWave Studio (MWS [4]) for this purpose and a first antenna model made of a single strap inside a box was included. The strap is fed through the upper port located at the helicon source side. The antenna is fully immersed into the loading medium (plasma or homogeneous dielectric) and the curved strap front face is aligned with the magnetic surfaces to simplify the modelling. The initial design of this antenna has been studied with MWS in the presence of homogeneous dielectric. The presence of a back wall will be discussed.
\end{abstract}

\section{INTRODUCTION}

The experimental device IShTAR [1,2] (Ion cyclotron Sheath Test ARrangement) is a linear test stand whose purpose is the study and characterisation of electric fields in front of Ion Cyclotron Range of Frequency (ICRF) antennas in magnetised plasmas. RF sheaths generated in front of ICRF antennas can induce deleterious effects like impurity generation, hot spots and sputtering, and their understanding is of prime importance. IShTAR allows for dedicated experiments and measurements on RF sheaths, and its simple geometry will facilitate direct comparisons with theory and modelling. The plasma is generated externally with an antenna that should allow us to reach the helicon transition. In the present work we will discuss two important aspects of the modelling: simulation of the magnetic configuration and the design and the electromagnetic modelling of the accepted option for an ICRF antenna. It was clear from the beginning of this activity that the antenna should be kept simple and easy to mount. A single-strap antenna has been considered. We will describe the various options considered (geometry and location inside the vessel), then geometry details will be provided and the results of a first optimisation will be presented.

\section{MAGNETIC CONFIGURATION MODELLING AND FIRST OPTIMISATION}

A parameterized magnetostatic model of the magnetic configuration was created with the AC/DC module of the COMSOL Multiphysics commercial software [3] in 3D and in axisymmetric 2D whenever possible. The general 3D model considers the two non-axial sets of coils in the present magnetic configuration [1]. The first set of coils is composed of two fixed and heavy former W7-A toroidal field coils $(r=0.55 \mathrm{~m}, h=0.4 \mathrm{~m}, b=0.183 \mathrm{~m}$, \#turns $=25$, section 


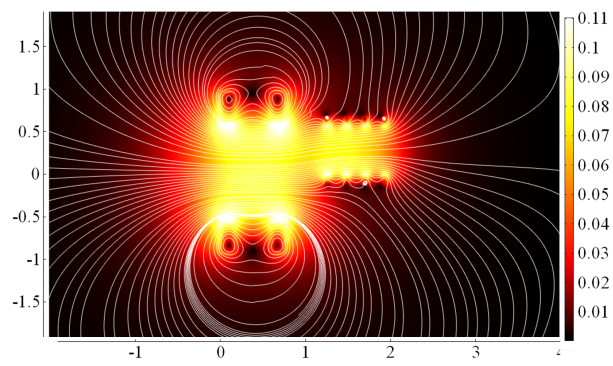

(a)

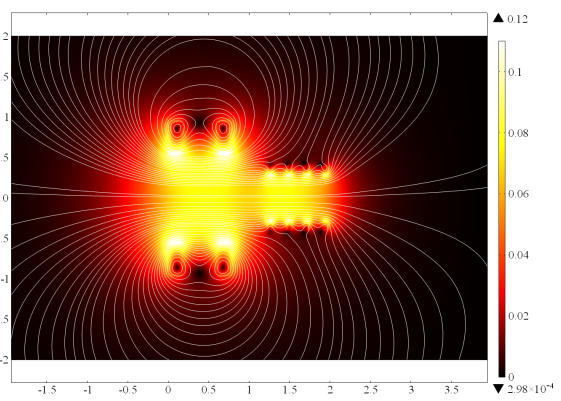

(b)

FIGURE 1. Magnetic field magnitude at the equatorial plane $(y=0)$ for $\mathrm{a})$ the present bi-axial configuration and $\mathrm{b})$ an axisymmetric configuration. The magnetic field lines are also shown. A deviation can clearly be seen on the bi-axial configuration.

of copper $\left.=1331 \mathrm{~mm}^{2}\right)$ that can be supplied with a maximum current of $8 \mathrm{kA}$ for $10 \mathrm{~s}$. The distance $(\mathrm{d}=0.42 \mathrm{~m})$ between both coils is not exactly the one expected from a Helmholtz coils configuration. 4 former spare coils of WEGA (a fifth could be possibly added) composed the second set of coils $(r=0.285 \mathrm{~m}, h=0.11 \mathrm{~m}, b=0.05 \mathrm{~m}$, \#turns $=13$, section of copper $=316 \mathrm{~mm}^{2}$ ) around the plasma source. They are mounted with a $0.175 \mathrm{~m}$ gap between coils. Additional degrees of freedom are provided by adjusting their position along the axis and rotation around the vertical axis. The present power supply for these coils allows for currents up to $1 \mathrm{kA}$. The two sets of coils are separated by a $0.455 \mathrm{~m}$ gap. The two coils axes are presently separated radially by a distance of $0.26 \mathrm{~m}$ due to the use of an existing flange without a central port to connect both vessels. From this model with a bi-axial magnetic configuration, we can infer that approximately $2.4 \mathrm{kA}$ are required in the 2 main large coils of IShTAR for $1 \mathrm{kA}$ in the 4 small coils to generate "smooth" magnetic field along field lines. The magnetic field configuration and field lines are shown in figure 1a in a horizontal plane. The magnitude of the magnetic field along the plasma source axis is 0.06T. A magnetic well exists between both sets of coils and its minimum reads $0.05 \mathrm{~T}$. This model notably reproduces the magnetic field lines deviation at the center of the main vessel and the ripples observed qualitatively during experiments. This deviation is characteristic of the bi-axial property of the configuration. It also breaks the invariance by rotation expected from each set of coils individually. Moreover, it is possibly linked to the instability that was observed at power beyond $1.3 \mathrm{~kW}$ [1]. The additional degree of freedom provided by the possibility to rotate the former WEGA coils independently does not seem necessary as it does not bring any useful changes. Actually, rotation causes the magnetic configuration to deviate even more from an axial configuration.

The advantages of centering the plasma source and sharing a common axis for both vessels are clearly shown on figure $1 \mathrm{~b}$. The deviation of the central field lines has disappeared. The invariance by rotation has been recovered. The best current ratio between both sets of coils to obtain a "smooth" magnetic field is 2.25 . The magnitude of the magnetic field along the magnetic axis is 0.077T. The magnetic profile along the axis still presents a well with a minimum of 0.068T. This simpler magnetic configuration allows a 2D axisymmetric model consuming much less computational resources (a few minutes and 10Go RAM in 3D compared to a few seconds and 1Go RAM in axisymmetric 2D). A comparison between both models yields the same solution when the 3D configuration can be reduced to 2D by assuming invariance by rotation. Manufacturing a new flange with a central port has been decided.

Further magnetic optimization is being attempted by this $2 \mathrm{D}$ model by adjusting the position of the former WEGA coils, adding a fifth coil. A possible solution to suppress the magnetic well in between both sets of coils is to reduce the distance to $0.25 \mathrm{~cm}$. The magnetic field is smoother over a longer distance without any magnetic field well, but the magnetic field decreases faster over the plasma source. However, a good homogeneity of the magnetic field over the plasma source seems to facilitate the transition to helicon mode and thus seems beneficial for obtaining high plasma density [1]. The decrease of the magnetic field magnitude can be attenuated by adding a fifth coil close to the most exterior former WEGA coil. Other kinds of optimization are currently being explored. 


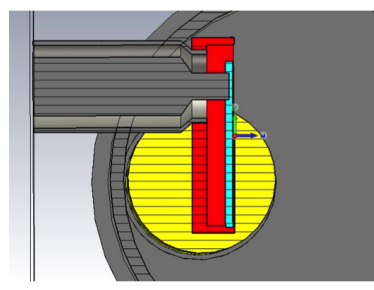

(a)

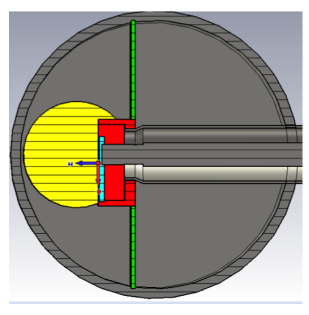

(b)

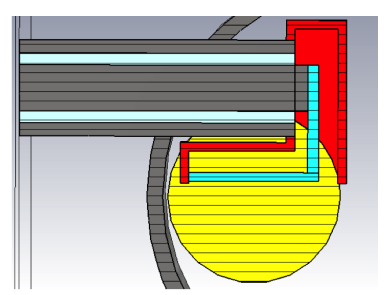

(c)

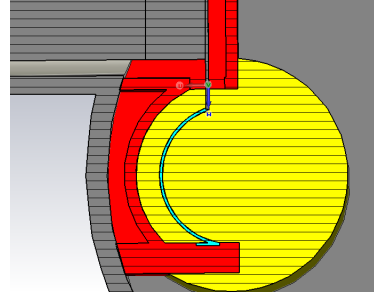

(d)

FIGURE 2. Various design philosophies successively considered for the IShTAR ICRF antenna: cut view also showing the plasma/dielectric assumed cylindrical centered on the plasma source.

\section{ICRF ANTENNA}

\section{Antenna design and location}

A fundamental ingredient to achieve the main mission of the IShTAR experiment is a simple but relevant ICRF antenna. For the first phase a single-short strap antenna inside a rectangular box connected to the vessel and fed by a coaxial line has been considered. Before designing this antenna in detail different options must be investigated to cope with the limited number and positions of the ports, the limited plasma volume, the constraints of being as close as possible to "real" tokamak conditions, and the expected simplicity of this first step design:

1. figure 2a: antenna strap on the wall, possibly rotatable around the coaxial axis, mounted at the port that is located on the same side as the plasma source; the antenna would mostly radiate in vacuum due to the limited volume occupied by the plasma and the fact that the E-field is maximal near the top gap;

2. figure 2b: vertical antenna strap located in the middle of the vessel, with an integrated wall, connected from the opposite "equatorial port"; this option is also not acceptable as it would require a very involved manufacturing work. In addition, the magnetic field gradient will be opposite to the tokamak case (high field side on the antenna).

3. figure 2c: horizontal antenna would make it possible to "dive" the antenna in the plasma. But in this case, the magnetic field gradient is all along the strap.

4. figure 2d: curved strap antenna following the cylindrical plasma shape completely emerged in the plasma, at a fixed magnetic field value and plasma density.

Although it is a bit more difficult to manufacture, the latter design was selected as its inherent symmetry greatly simplifies the modelling efforts. This is an important aspect of the test facility compared to an existing fusion machine.

\section{Detailed design \& first optimisation}

The Microwave Studio (MWS, [4]) model of the currently retained geometry (with initial dimensions in $\mathrm{mm}$ ) of the IShTAR antenna is visible in figure 3. The strap is fed from the top feedthrough by a coaxial transmission line connected to a vertical plate inserted into the strap. Unlike usual ICRF antennas the IShTAR antenna is not designed to couple as much power as possible to the plasma, but rather to generate the typical electric fields structures expected in a tokamak. We will therefore start with a "reasonable" geometry which will be further adapted or optimised in the coming years on basis of the first experimental results. Simulations were performed with a dielectric loading (relative permittivity $=500$ ). Plasma simulations were also performed but did not lead to converged results (also with COMSOL). This issue will be investigated in the near future.

The first simulations with MWS clearly demonstrated some issues due to the presence of sharp angles in the lateral limiters. Even by further smoothing these angles (see 3c) the electric fields maps were dominated by peaked radiations at the level of the limiters, which could also be partly due to numerical issues (meshing). We therefore decided to replace these profiled limiters by rectangular plates: see figure 3d, resulting in a more regular electric field distribution. This change also considerably simplifies the building of the antenna box. Figure 4 shows the magnitude of the electric field at $10 \mathrm{MHz}$ for dielectric loading and a common normalisation of the fields corresponding to a strap short-circuit 


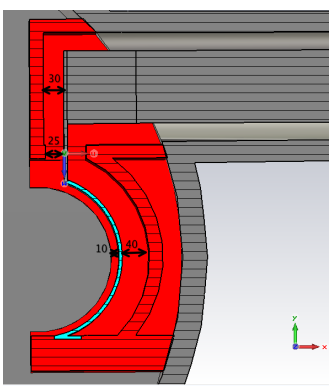

(a)

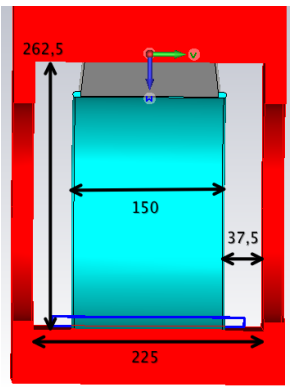

(b)

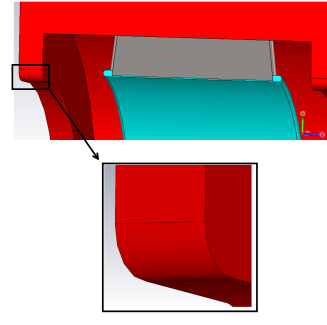

(c)

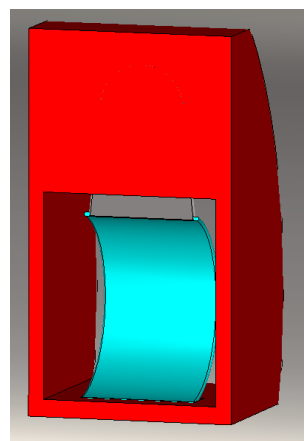

(d)

FIGURE 3. MWS model of the IShTAR ICRF antenna and its feeding line; (a) lateral cut view of the initial geometry; (b) front view; (c) zoom of the angular part of the limiter; (d) simplified geometry; All dimensions are given in $\mathrm{mm}$

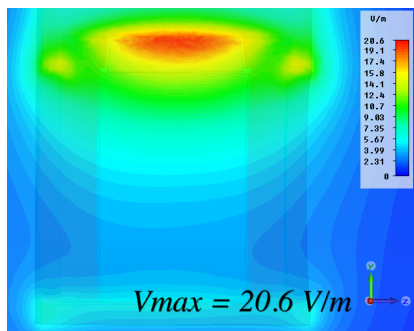

(a)

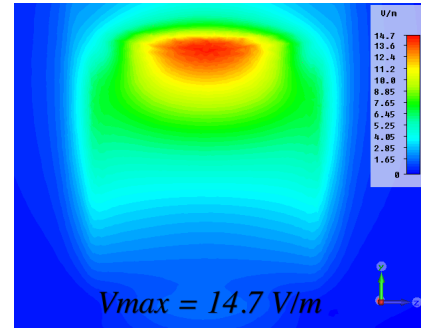

(b)

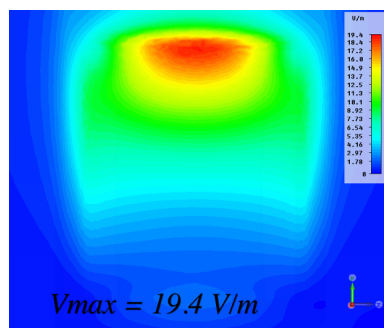

(c)

FIGURE 4. Magnitude of the electric field at $10 \mathrm{~mm}$ from the antenna mouth; (a) initial model (b) simplified model with back wall (c) simplified model without back wall

current of 1A. The last case corresponds to a geometry where the curved back wall was removed from the antenna box. The larger self-inductance of the latter geometry leads to an increase of the electric fields and voltages (for a constant current). It is therefore better to not consider such a back wall in the current design.

\section{CONCLUSIONS}

The IShTAR device is a very promising tool for the study of RF-induced sheaths in front of ICRF antennas in tokamak plasmas. In the present work various aspects of its design were investigated. On the one hand the multi physics code COMSOL was used to analyse the magnetic configuration and to optimise the smoothness of the magnetic field. The advantages of centering the plasma source and sharing a common axis for both vessels was clearly demonstrated. On the other hand an initial proposal for an ICRF antenna design was studied with MWS. In particular the effect of the antenna box geometry was studied to improve electric fields distribution and increase their amplitude.

\section{Aknowledgement}

This work has been carried out within the framework of the EUROfusion Consortium and has received funding from the Euratom research and training programme 2014-2018 under grant agreement No 633053. The views and opinions expressed herein do not necessarily reflect those of the European Commission.

\section{REFERENCES}

1. R. D'Inca et al., IshTAR: a test facility to investigate sheaths effects during Ion Cyclotron Resonance Heating, to be submitted for publication to Fusion Engineering and Design

2. K. Crombé et al., Studies of RF sheaths and diagnostics on IShTAR, invited paper at this conference

3. R.W. Pryor. Multiphysics modeling using COMSOL: a first principles approach. Jones and Bartlett Publishers, 2011

4. CST STUDIO SUITE®, CST AG, Germany, www.cst.com. 